\title{
Incipient Sensor Fault Detection for Inverter Devices in Electric Railway Traction Systems
}

\author{
Kangkang Zhang*, Bin Jiang*, Xing-gang Yan ${ }^{\dagger}$, Yajie $\mathrm{Ma}^{*}$, \\ *College of Automation Engineering \\ Nanjing University of Aeronautics and Astronautics, Nanjing 210016, China \\ Email: KangZhang359@163.com; binjiang@ nuaa.edu.cn; yajie030410331@126.com \\ ${ }^{\dagger}$ School of Engineering and Digital Arts \\ University of Kent, Canterbury, Kent CT2 7NT, United Kingdom \\ Email: x.yan@kent.ac.uk
}

\begin{abstract}
This paper proposes an incipient sensor fault detection method for three-phase PWM inverter devices in electric railway traction systems. An adaptive and sliding mode unknown input observer is designed for sensor faulty inverter system. The invariant ellipsoid is used to generate threshold. The parameters of the observer are particularly designed such that the estimation errors converge to the threshold invariant ellipsoid before the sensor fault develops to incipient fault degree, and the estimation errors exceed the threshold after the sensor fault develops to incipient fault degree. Finally, simulations based on the traction system in CRH2 (China Railway High-speed) are presented to verify the effectiveness of the proposed method.

Index Terms-Incipient fault detection, adaptive sliding mode estimator, invariant ellipsoid.
\end{abstract}

\section{INTRODUCTION}

Safety is the first concern in high-speed railway operation, which is greatly dependent on the reliability of information control systems of high-speed trains. The traction drive subsystem is the core of information control systems in high-speed train systems, which plays an important role in electric railway running safety. Therefore, the fault diagnosis and FTC (faulttolerant control) mechanism are necessary for modern highspeed railway systems, especially for the traction subsystems.

Modern railway traction power systems are fed by $2 x$ $25 \mathrm{KV} / 50 \mathrm{~Hz}$ single phase ac current sources in [1] or by $1500 \mathrm{~V}$ dc voltage from electric railway substations in [2]. A typical ac/dc/ac power system used for electrical traction drives is shown in Fig. 1 (see, e.g. [3]), which includes a catenary, a voltage transformer, a single phase PWM rectifier, a three-phase PWM inverter and driving motors. In the traction systems, the electric power is transmitted to the drive motors through pantograph, voltage transformers, single phase PWM rectifiers and three-phase PWM inverters. The inverter is driven by the dc link voltage, provided by the rectifier, while the driving motors are driven directly by the three-phase PWM inverter which affects the motion performance of the driving

This work is supported in part by the National Natural Science Foundation of China (Grant 61490703 and 61573180), the Project Funded by the Priority Academic Program Development of Jiangsu Higher Education Institutions, Fundamental Research Funds for the Central Universities (NO. NE2014202) and the Research Innovation Program for College Graduates of Jiangsu Province (KYLX16_0374)

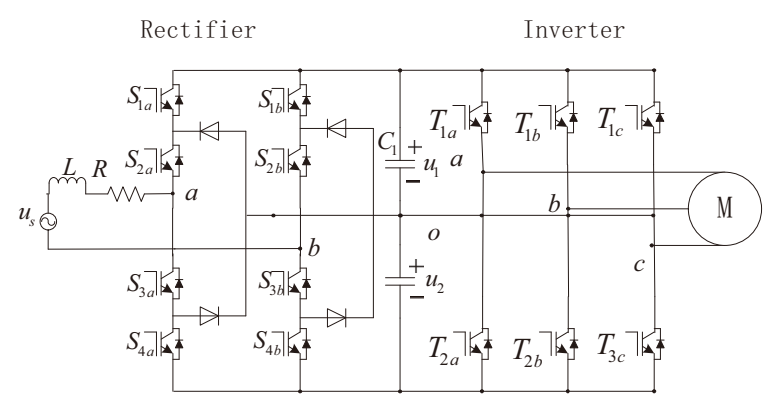

Fig. 1. Railway traction circuit schematic diagram.

motors greatly. Over a long period of time, aging components, such as electrolyte loss effectiveness of electrolytic capacitors, in the current sensors and voltage sensors, may deduce incipient faults, and further develop to serious failures, which would degrade performance of the total traction systems seriously. Therefore, early incipient sensor fault diagnosis is significant.

During the past several decades, there are many results about the incipient fault detection using adaptive technique and sliding mode technique, such as [5], [6] and [7], [8]. Different adaptive technique based fault estimation modules and sliding mode technique based fault reconstruction modules are proposed to estimate the fault parameters in these papers. However, it is still very challenging to apply these approaches to detect incipient faults, especially in the presence of disturbances and uncertainties. In [9] and [10], an invariant ellipsoid method is proposed to deal with $\mathcal{L}_{\infty}$ disturbances, which motivates us to combine the adaptive technique, sliding mode technique and invariant ellipsoid method to detect incipient faults. However, in inverter systems, there are observer unmatched unknown inputs which cannot be compensated through output channels directly. Therefore, an robust detection observer should be designed for the inverter system.

In this paper, an adaptive and sliding mode unknown input observer is designed such that the fault estimation errors converge to a designed invariant ellipsoid before the sensor fault developing to incipient fault degree. Moreover, after the fault develops to incipient fault degree, the estimation errors are guaranteed to exceed the invariant ellipsoid in finite time. 
Correspondingly, the sensor incipient fault detection decision schemes are also developed.

\section{Problem Formulation}

\section{A. Dynamic Modeling of Inverter}

The topology structure of the inverter device used in the CRH2's traction system is shown in Fig. 2, where $L_{f}, C_{f}$ and $r$ are the filter inductor, capacitor and equivalent resistance, respectively, $V_{d c}$ is the dc voltage source, $v_{j n}, j=a, b, c$ are the inverter bridge voltages, $v_{o j}$ and $i_{o j}, j=a, b, c$ are the load voltages and currents, respectively.

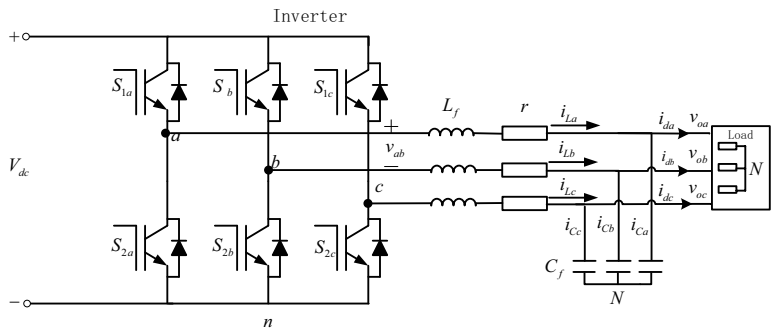

Fig. 2. Three-phase PWM inverter topology

In electrical traction systems, the motors' speeds are regulated by varying the ac voltage frequencies in inverter devices. However, the frequencies vary more slowly than sampling time in practical transducers, that is $\dot{\omega} \approx 0$. Therefore, the $d-q$ coordinate transformation, which is always used in inverter devices with fixed frequencies, can also used to frequencies varying inverter devices in electrical traction systems. Let $x=\operatorname{col}\left(v_{o d}, v_{o q}, i_{L d}, i_{L q}\right)$ and $i_{0}=\operatorname{col}\left(i_{o d}, i_{o q}\right)$. Then, based on [11], the state-space model of the inverter device with sensor fault is described by

$$
\begin{aligned}
\dot{x} & =A x+B u+E i_{o}, \\
e_{y r} & =C x+F f-y_{r}
\end{aligned}
$$

where the control input $u$ in (1) and $i_{0}$ are measurable,

$$
\begin{gathered}
A=\left[\begin{array}{cccc}
0 & \omega & \frac{1}{C_{f}} & 0 \\
-\omega & 0 & 0 & \frac{1}{C_{f}} \\
-\frac{1}{L_{f}} & 0 & -\frac{r}{L_{f}} & \omega \\
0 & -\frac{1}{L_{f}} & -\omega & -\frac{r}{L_{f}}
\end{array}\right], B=\left[\begin{array}{cc}
0 & 0 \\
0 & 0 \\
\frac{V_{d c}}{L_{f}} & 0 \\
0 & \frac{V_{d c}}{L_{f}}
\end{array}\right], \\
E=\left[\begin{array}{cc}
-\frac{1}{C_{f}} & 0 \\
0 & -\frac{1}{C_{f}} \\
0 & 0 \\
0 & 0
\end{array}\right], C=\left[\begin{array}{llll}
1 & 0 & 0 & 0 \\
0 & 1 & 0 & 0
\end{array}\right],
\end{gathered}
$$

where the angular velocity $\omega$ of the inverter device in electrical system belong to $[100,1382]$. And the $F$ is the fault distribution matrix which is assumed to be full column rank throughout the paper.
In this case, the polytopic representation can be written as follows:

$$
\begin{aligned}
\dot{x} & =\sum_{i=1}^{2} \zeta_{i}(\omega) A_{i} x+B u+E i_{0}, \\
e_{y r} & =C x+F f-y_{r}
\end{aligned}
$$

The obtained system evolves in a polytope of two vertices corresponding to the extreme values of the angular velocity $\omega$. Thus, the matrices $A_{i}$ are given by

$$
A_{1}=\left[\begin{array}{cccc}
0 & 100 & \frac{1}{C_{f}} & 0 \\
-100 & 0 & 0 & \frac{1}{C_{f}} \\
-\frac{1}{L_{f}} & 0 & -\frac{r}{L_{f}} & 100 \\
0 & -\frac{1}{L_{f}} & -100 & -\frac{r}{L_{f}}
\end{array}\right], A_{2}=\left[\begin{array}{cccc}
0 & 1382 & \frac{1}{C_{f}} & 0 \\
-1382 & 0 & 0 & \frac{1}{C_{f}} \\
-\frac{1}{L_{f}} & 0 & -\frac{r}{L_{f}} & 1382 \\
0 & -\frac{1}{L_{f}} & -1382 & -\frac{r}{L_{f}}
\end{array}\right] .
$$

And the weighting functions $\zeta_{i}(\omega)$ are given by

$$
\zeta_{1}(\omega)=\frac{\omega-100}{1282} \text { and } \zeta_{2}(\omega)=\frac{1382-\omega}{1282} .
$$

Let $A(\omega)=\sum_{i=1}^{2} \zeta_{i}(\omega) A_{i}$ for the describing simplicity in the sequel.

\section{B. Incipient Sensor Fault Modeling}

Since incipient faults are small in amplitude, piecewise continuous and develop slowly, they can be modeled based on the following lemma.

Lemma 1: [12] For any piecewise continuous vector function $f: \mathcal{R}^{+} \rightarrow \mathcal{R}^{q}$, and a stable $q \times q$ matrix $A_{f}$, there always exists an input vector $\xi \in \mathcal{R}^{q}$ such that $\dot{f}=A_{f} f+\xi$. From Lemma 1, incipient faults $f(t)$ can be modeled by

$$
\dot{f}=A_{f} f+\xi, f(0)=0
$$

where $A_{f}$ is a stable matrix with appropriate dimensions, and $\xi=\left[\xi_{1}^{T}, \cdots, \xi_{q}^{T}\right]^{T} \in \mathcal{R}^{q}$ is an unknown vector. Taking the Laplace transformation on Eq. (5), it is clear to see that in the frequency domain, $f(s)=\left(s I-A_{f}\right)^{-1} \xi$, which shows that the fault signal $f$ is determined by $\xi$ completely. Let $x_{a}:=$ $\operatorname{col}(x, f)$. System (3) and incipient sensor faults (5) can be represented in an augmented form

$$
\begin{aligned}
& \dot{x}_{a}=A_{a}(\omega) x_{a}+B_{a} u+E_{a} i_{0}+E_{a} d+D_{a} \xi, \\
& e_{y r}=C_{a} x_{a}-y_{r}
\end{aligned}
$$

where

$$
\begin{gathered}
A_{a}=\left[\begin{array}{ll}
A(\omega) & \\
& A_{f}
\end{array}\right] \in \mathcal{R}^{(n+q) \times(n+q)}, B_{a}=\left[\begin{array}{c}
B_{0} \\
0
\end{array}\right] \in \mathcal{R}^{(n+q) \times m}, \\
E_{a}=\left[\begin{array}{c}
B_{0} \\
0
\end{array}\right] \in \mathcal{R}^{(n+q) \times h}, D_{a}=\left[\begin{array}{c}
0 \\
I_{q}
\end{array}\right] \in \mathcal{R}^{(n+q) \times q}, C_{a}= \\
{\left[\begin{array}{cc}
C & F
\end{array}\right] \in \mathcal{R}^{p \times(n+q)} \text {. Suppose that } F \text { in (3) has the form }} \\
F=\left[\begin{array}{c}
0_{(p-q) \times q} \\
I_{q}
\end{array}\right] .
\end{gathered}
$$

Then $\operatorname{rank}\left(C_{a} D_{a}\right)=q$ and $\operatorname{rank}\left(D_{a}\right)=q$, which implies the relative degree of the triple $\left(A_{a}, D_{a}, C_{a}\right)$ is inherently one.

To reject the bounded exogenous disturbances, the invariant ellipsoid concept is introduced. 
Definiton 1: The ellipsoid

$$
\varepsilon(P)=\left\{x: x^{T} P x<1\right\}, P>0
$$

with the center in the origin and a radius matrix $P$, is said to be an invariant ellipsoid for the systems $\dot{x}=A x+D \omega$ with respect to the bounded disturbances $\omega$

- if $x(0) \in \varepsilon(P)$, then $x(t) \in \varepsilon(P)$ for all $t \geq 0$;

- and if $x(0) \notin \varepsilon(P)$, then $x(t) \rightarrow \varepsilon(P)$ for $t \rightarrow \infty$.

\section{Incipient Fault Description}

In this paper, a worst-case scale variable between the influence of faults and the influence of disturbances is proposed to define the incipient faults. First of all, a regulate output $z$ is given, which can effectively feedback on faults and disturbances. Then two incremental quantities are defined by

$$
\Delta_{z_{f}}=G_{f}(f), \Delta_{z_{d}}=G_{d}(d)
$$

where $G_{f}(\cdot)$ and $G_{d}(\cdot)$ represent functions that from faults $f$ and disturbances $d$ to $z$ respectively. Then the scale variable is defined by

$$
\Gamma=\inf \frac{\left\|\Delta_{z_{f}}\right\|_{p}}{\left\|\Delta_{z_{d}}\right\|_{p}}=\inf \frac{\left\|G_{f}(f)\right\|_{p}}{\left\|G_{d}(d)\right\|_{p}} .
$$

where $\inf (\cdot)$ represents the minimal value.

Therefore, based on (10), the developing process of a fault is divided into three degree which is shown as follows.

(a) when $0 \leq \Gamma<\underline{\Gamma}$, the fault is unnecessary to detect;

(b) when $\underline{\Gamma}<\Gamma<\bar{\Gamma}$, the fault is in an incipient fault degree;

(c) When $\underline{\Gamma}<\Gamma<+\infty$, the fault is in a serious fault degree.

Suppose that $T_{0}$ is the time that the fault develops to incipient fault degree, and that there exist a positive definite matrix $Q_{d} \in \mathcal{R}^{h \times h}$ and a positive constants $d_{0}$ and $d_{1}$ throughout the paper such that

$$
d_{1}<d^{T} Q_{d} d \leq d_{0} .
$$

Then the following two assumptions about the amplitudes of faults and disturbances are presented.

Assumption 1: Before the fault develops to incipient fault degree $\left(t<T_{0}\right)$, the fault parameters $\xi, \dot{\xi}$ and disturbance $d$ satisfy that

$$
\xi^{T} Q_{\xi} \xi \leq \Gamma_{0} d^{T} Q_{d} d, \dot{\xi}^{T} Q_{\xi} \dot{\xi} \leq \Gamma_{0} d^{T} Q_{d} d,
$$

and after the fault develops to incipient fault degree $\left(t>T_{0}\right)$, there exist two constants $\underline{\Gamma}$ and $\bar{\Gamma}$ satisfying $\Gamma_{0} \leq \underline{\Gamma} \leq \bar{\Gamma}$ such that

$$
\underline{\Gamma} d^{T} Q_{d} d \leq \xi^{T} Q_{\xi} \xi \leq \bar{\Gamma} d^{T} Q_{d} d, \underline{\Gamma} d^{T} Q_{d} d \leq \dot{\xi}^{T} Q_{\xi} \dot{\xi} \leq \bar{\Gamma} d^{T} Q_{d} d
$$

where $Q_{\xi} \in \mathcal{R}^{q \times q}$ and $Q_{\xi} \in \mathcal{R}^{q \times q}$ are positive definite matrices

\section{Incipient Fault Detection Observer Design}

Based on [13], since the relative degree of the triple $\left(A_{a}(\omega), D_{a}, C_{a}\right)$ is one, there exists a coordinate transformation for augmented system (6) such that the triple $\left(A_{a}(\omega), D_{a}, C_{a}\right)$ in the new coordinates can be described by

$\left(\left[\begin{array}{c|cc}A_{a 11}(\omega) & \multicolumn{2}{|c}{A_{a 12}(\omega)} \\ \hline A_{a 211}(\omega) & A_{a 22}^{11}(\omega) & A_{a 22}^{12}(\omega) \\ \hline A_{a 212}(\omega) & A_{a 22}^{21}(\omega) & A_{a 22}^{22}(\omega)\end{array}\right],\left[\begin{array}{c}0 \\ D_{a 2}\end{array}\right],\left[\begin{array}{ll}0 & C_{a 2}\end{array}\right]\right)$

where $A_{a 11}(\omega) \in \mathcal{R}^{(n+q-p) \times(n+q-p)}, C_{a 2} \in \mathcal{R}^{p \times p}$ is orthogonal and $D_{a 2}=\left[0, D_{a 22}^{T}\right]^{T}$ with $D_{a 22} \in \mathcal{R}^{q \times q}$ being nonsingular. Denote $x_{a}=\operatorname{col}\left(x_{1}, x_{2}\right)$ with $x_{1} \in \mathcal{R}^{n+q-p}$ and $x_{2} \in \mathcal{R}^{p}$. Then, based on [13], the system (6) can be transformed to

$$
\begin{aligned}
\dot{x}_{1}= & A_{a 11}(\omega) x_{1}+A_{a 12}(\omega) x_{2}+B_{a 1} u+E_{a 1} i_{0}+E_{a 1} d, \\
\dot{x}_{21}= & A_{a 211}(\omega) x_{1}+A_{a 22}^{11}(\omega) x_{21}+A_{a 22}^{12}(\omega) x_{22}+B_{a 21} u \\
& +E_{a 21} i_{0}+E_{a 21} d, \\
\dot{x}_{22}= & A_{a 212}(\omega) x_{1}+A_{a 22}^{21}(\omega) x_{21}+A_{a 22}^{22}(\omega) x_{22}+B_{a 22} u \\
& +E_{a 22} i_{0}+E_{a 22} d+D_{a 22} \xi, \\
e_{y r}= & C_{a 2} x_{2}-y_{r},
\end{aligned}
$$

where $x_{2}=\operatorname{col}\left(x_{21}, x_{22}\right), B_{a 1}$ and $B_{a 2}$ are obtained from [13].

By a linear coordinate transforming $z=T x_{a}$ where $z:=$ $\operatorname{col}\left(z_{1}, z_{21}, z_{22}\right)$ with $z_{1} \in \mathcal{R}^{n+q-p}, z_{21} \in \mathcal{R}^{p-q}$ and $z_{22} \in \mathcal{R}^{q}$, and

$$
T=\left[\begin{array}{cc}
I_{n+q-p} & L \\
0 & I_{q}
\end{array}\right]
$$

where $L=\left[L_{1}, 0_{(n+q-p) \times q}\right]$ with $L_{1} \in \mathcal{R}^{(n+q-p) \times(p-q)}$, then $z_{1}=$ $x_{1}+L_{1} x_{21}$ and the system (15) becomes

$$
\dot{z}_{1}=\hat{A}_{a 11}(\omega) z_{1}+\hat{A}_{a 12}(\omega) z_{2}+\hat{B}_{1} u+\hat{E}_{1} i_{0}+\hat{E}_{1} d,
$$

where matrices $\hat{A}_{a 11}(\omega)=A_{a 11}(\omega)+L A_{a 21}(\omega)$ is Hurwitz, $\hat{A}_{12}=$ $-\left(A_{a 11}+L A_{a 21}\right) L+\left(A_{a 12}+L A_{a 22}\right), \hat{B}_{1}=B_{a 1}+L B_{a 2}$ and $\hat{E}_{1}=$ $E_{a 1}+L E_{a 2}$.

For subsystems (20) and (16)-(18), an adaptive and sliding mode unknown input observer is proposed as

$$
\begin{aligned}
\dot{\hat{z}}_{1}= & \hat{A}_{a 11}(\omega) \hat{z}_{1}+\hat{A}_{a 12}(\omega) x_{2}+K_{1}\left(x_{22}-\hat{z}_{22}\right)+\hat{B}_{1} u+\hat{E}_{1} i_{0}, \\
\dot{z}_{21}= & A_{a 211}(\omega) \hat{z}_{1}+A_{a 22}^{11}(\omega) \hat{z}_{21}+K_{21}\left(x_{21}-\hat{z}_{21}\right)+A_{a 22}^{12}(\omega) x_{22} \\
& +B_{a 21} u+E_{a 21} i_{0}+E_{a 21} d+v \\
\dot{z}_{22}= & A_{a 212}(\omega) \hat{z}_{1}+A_{a 22}^{21}(\omega) x_{21}+A_{a 22}^{22}(\omega) \hat{z}_{22}+K_{22}\left(x_{22}-\hat{z}_{22}\right) \\
& +B_{a 22} u+E_{a 22} i_{0}+E_{a 22} d+D_{a 22} \hat{\xi}, \\
\dot{\hat{\xi}}= & \Gamma D_{a 22}^{T} P_{22}\left(x_{22}-\hat{z}_{22}\right)-\sigma \Gamma \hat{\xi}, \\
\hat{e}_{y r}= & \hat{z}_{2}-y_{r}
\end{aligned}
$$

where the gain matrix $K_{1}$ is chosen as $K_{1}=$ $\left.\hat{A}_{a 12}(\omega) \operatorname{diag}\left\{0_{(p-q) \times(p-q)}\right\}, I_{q}\right)+G_{1}(\omega)$ with $G_{1}(\omega)=$ $\sum_{i=1}^{N} \zeta(\omega) G_{1 i} \in \mathcal{R}^{(n+q-p) \times q}, \quad K_{21}=\hat{A}_{21}(\omega)-G_{21}$ and $K_{22}=\hat{A}_{22}(\omega)+G_{22}(\omega)$ with $G_{21} \in \mathcal{R}^{(p-q) \times(p-q)}$ and $G_{22}(\omega)=\sum_{i=1}^{N} \zeta(\omega) G_{22 i} \in \mathcal{R}^{q \times q}$. The matrix $G_{21 i}$ is symmetric negative definite and Hurwitz. The gain matrices $G_{1 i}, G_{22 i}$, the constant $\sigma>0$ and weighting matrix $\Gamma=\Gamma^{T}>0$ 
will be optimized in the sequel. The nonlinear function $v$ is given by

$$
v=\rho(\cdot) \operatorname{sgn}\left(x_{21}-\hat{z}_{21}\right)
$$

where the scalar function $\rho(\cdot)$ is determined later.

Let $e_{1}=z_{1}-\hat{z}_{1}, e_{21}=x_{21}-\hat{z}_{21}, e_{22}=x_{22}-\hat{z}_{22}$ and $e_{\xi}=\xi-\hat{\xi}$. Denote $e_{2}=\operatorname{col}\left(e_{21}, e_{22}\right)$. Then by comparing (20) and (16)(18) with (21)-(24), the error dynamical system is obtained by

$$
\begin{aligned}
\dot{e}_{1} & =\hat{A}_{a 11}(\omega) e_{1}-G_{1}(\omega) e_{22}+\hat{E}_{1} d, \\
\dot{e}_{21} & =A_{a 211}(\omega) e_{1}+G_{21} e_{21}+E_{a 21} d-v, \\
\dot{e}_{22} & =A_{a 212} e_{1}-G_{22}(\omega) e_{22}+E_{a 22} d+D_{a 22} e_{\xi}, \\
\dot{\hat{\xi}} & =\Gamma D_{a 22}^{T} P_{22}\left(x_{22}-\hat{z}_{22}\right)-\sigma \Gamma \hat{\xi} .
\end{aligned}
$$

where $P_{22}$ is the Lyapunov matrix of subsystem (29).

Since the relative degree of the triple the triple $\left(A_{a}(\omega), D_{a}, C_{a}\right)$ is one, then from [13], there exist a constructed the constraint Lyapunov matrix

$$
P:=\left[\begin{array}{cc}
P_{1} & P_{1} L \\
L^{T} P_{1} & P_{2}+L^{T} P_{2} L
\end{array}\right]
$$

where $P_{1} \in \mathcal{R}^{(n+q-p) \times(n+q-p)}$ and $P_{2} \in \mathcal{R}^{p \times p}$, such that

$$
\left(T^{-1}\right)^{T} P T^{-1}=\left[\begin{array}{ll}
P_{1} & \\
& P_{2}
\end{array}\right]
$$

where $P_{1}$ is a Lyapunov matrix of subsystem (27), and $P_{2}$ is a Lyapunov function of subsystem (28)-(29).

Since (28) and (29) are decoupled, the Lyapunov matrix of subsystem (28)-(29) is a diagonal matrix. Thus, the Lyapunov matrix $P_{2}$ is a block diagonal matrix, which can be chosen as $P_{2}=\operatorname{diag}\left\{P_{21}, P_{22}\right\}$ with $P_{21}$ and $P_{22}$ being the Lyapunov matrix respectively.

Consider the ellipsoid

$\varepsilon(\mathscr{P})=\left\{\operatorname{col}\left(e_{1}, e_{22}, e_{\xi}\right): \operatorname{col}\left(e_{1}, e_{22}, e_{\xi}\right)^{T} \mathscr{P} \operatorname{col}\left(e_{1}, e_{22}, e_{\xi}\right)<1\right\}$

where $\mathscr{P}=\operatorname{diag}\left\{P_{1}, P_{22}, \Gamma^{-1}\right\}>0$. Then the following theorem is ready to be presented.

Proposition 1: Under Assumption 1, for certain $\sigma>0$ and some $\tau_{01} \geq \tau_{02}>0$ and $\tau_{11} \geq \tau_{12}>0$, the set $\varepsilon(\mathscr{P})$ is an invariant ellipsoid for error system (27) and (29)-(30), if there exist SPD matrices $P_{1} \in \mathcal{R}^{(n+q-p) \times(n+q-p)}, P_{22} \in \mathcal{R}^{p \times p}$ and $\Gamma^{-1} \in \mathcal{R}^{q \times q}$, matrices $Y \in \mathcal{R}^{(n+q-p) \times p}, W_{1 i} \in \mathcal{R}^{(n+q-p) \times q}$ and $W_{2 i} \in \mathcal{R}^{p \times p}$ and scalar $\sigma$ such that

$$
\begin{gathered}
P_{22}>0, P_{1}>0, \Gamma^{-1}>0, \sigma>0 \\
{\left[\begin{array}{cccccc}
\Theta_{11}+\tau_{01} P_{1} & \Theta_{12} & 0 & 0 & 0 & \Theta_{16} \\
* & \Theta_{22}+\tau_{01} P_{22} & 0 & 0 & 0 & \Theta_{26} \\
* & * & -2 \sigma I+\tau_{01} \Gamma^{-1} & \sigma I & \Gamma^{-1} & 0 \\
* & * & * & -\frac{\tau_{02}}{\alpha d_{0}} Q_{\xi} & 0 & 0 \\
* & * & * & * & -\frac{\tau_{02}}{\alpha d_{0}} Q_{\xi} & 0 \\
* & * & * & * & * & -\frac{\tau_{02}}{\alpha d_{0}} Q_{d}
\end{array}\right]<0,} \\
{\left[\begin{array}{cccccc}
-\Theta_{11}-\tau_{11} P_{1} & -\Theta_{12} & 0 & 0 & 0 & -\Theta_{16} \\
* & -\Theta_{22}-\tau_{11} P_{22} & 0 & 0 & 0 & -\Theta_{26} \\
* & * & 2 \sigma I-\tau_{11} \Gamma^{-1} & -\sigma I & -\Gamma^{-1} & 0 \\
* & * & * & -\frac{\tau_{12}}{\beta d_{1}} Q_{\xi} & 0 & 0 \\
* & * & * & * & -\frac{\tau_{12}}{\beta d_{1}} Q_{\xi} & 0 \\
* & * & * & * & * & -\frac{\tau_{12}}{\beta d_{1}} Q_{d}
\end{array}\right]<0} \\
*
\end{gathered}
$$

where $\Theta_{11}=\operatorname{He}\left(P_{1} A_{a 11}(\omega)+Y A_{a 21}(\omega)\right), \Theta_{12}=-W_{1}(\omega)+$ $A_{a 212}^{T}(\omega) P_{22}, \Theta_{16}=P_{1} E_{a 1}+Y E_{a 2}, \Theta_{22}=-H e\left(W_{2}(\omega)\right), \Theta_{26}=$ $P_{22} E_{a 22}, \alpha=2 \Gamma_{0}+1$ and $\beta=2 \Gamma+1$. Then, the gain matrices $L_{1}=P_{1}^{-1} Y, G_{1 i}=P_{1}^{-1} W_{1 i}$ and $\bar{G}_{2 i}=P_{22}^{-1} W_{2 i}$.

Proof: The Lyapunov candidate function is chosen as $V=$ $e_{1}^{T} P_{1} e_{1}+e_{22}^{T} P_{22} e_{22}+e_{\xi}^{T} \Gamma^{-1} e_{\xi}$. Note that $\dot{e}_{\xi}=\dot{\xi}-\dot{\hat{\xi}}$. Then the time derivative of $V$ along the trajectory of subsystem (27) and (29)-(30) is

$$
\begin{aligned}
\dot{V}= & e_{1}^{T}\left(P_{1} \hat{A}_{a 11}(\omega)+\hat{A}_{a 11}(\omega)^{T} P_{1}\right) e_{1}-2 e_{1}^{T} P_{1} G_{1}(\omega) e_{22} \\
& +2 e_{1}^{T} P_{1} \hat{E}_{1} d+2 e_{22}^{T} P_{22} A_{a 212}(\omega) e_{1} \\
& -e_{22}^{T}\left(P_{22} G_{2}(\omega)+G_{2}(\omega)^{T} P_{22}\right) e_{22}+2 e_{22}^{T} P_{22} D_{a 22} e_{\xi} \\
& +2 e_{22}^{T} P_{22} E_{a 22} d-2 e_{\xi}^{T} D_{a 22}^{T} P_{22} e_{22}-2 \sigma e_{\xi}^{T} e_{\xi}+2 \sigma e_{\xi}^{T} \xi \\
& +2 e_{\xi}^{T} \Gamma^{-1} \xi \\
= & {\left[\begin{array}{c}
e_{1} \\
e_{22} \\
e_{\xi} \\
\xi \\
\dot{\xi} \\
d
\end{array}\right]\left[\begin{array}{cccccc}
\Xi_{11} & \Xi_{12} & 0 & 0 & 0 & \Xi_{16} \\
* & \Xi_{22} & 0 & 0 & 0 & \Xi_{26} \\
* & * & -2 \sigma I & \sigma I & \Gamma^{-1} & 0 \\
* & * & * & 0 & 0 & 0 \\
* & * & * & * & 0 & 0 \\
* & * & * & * & * & 0
\end{array}\right]\left[\begin{array}{c}
e_{1} \\
e_{22} \\
e_{\xi} \\
\xi \\
\xi \\
d
\end{array}\right] }
\end{aligned}
$$

where $\Xi_{11}=H e\left(P_{1} \hat{A}_{a 11}(\omega)\right), \Xi_{12}=-P_{1} G_{1}(\omega)+A_{a 212}^{T}(\omega) P_{22}$, $\Xi_{16}=P_{1} \hat{E}_{1}, \Xi_{22}=-H e\left(P_{22} G_{2}(\omega)\right)$ and $\Xi_{26}=P_{22} E_{a 22}$.

Obviously, the $\varepsilon(\mathscr{P})$ is an invariant ellipsoid if and only if $\dot{V}<0$, for any $\left(e_{1}, e_{22}, e_{\xi}\right)$ satisfying $\left(e_{1}, e_{22}, e_{\xi}\right)^{T} \mathscr{P}\left(e_{1}, e_{22}, e_{\xi}\right) \geq 1$ and for $\operatorname{col}(\xi, \dot{\xi}, d)$ satisfying Assumption 1.

From Assumption $1, \xi, \dot{\xi}$ and $d$ satisfy

$$
\frac{1}{\Gamma_{0} d_{0}}\left[\begin{array}{c}
e_{1} \\
e_{22} \\
e_{\xi} \\
\xi \\
\dot{\xi} \\
d
\end{array}\right]^{T}\left[\begin{array}{cccccc}
0 & 0 & 0 & 0 & 0 & 0 \\
* & 0 & 0 & 0 & 0 & 0 \\
* & * & 0 & 0 & 0 & 0 \\
* & * & * & Q_{\xi} & 0 & 0 \\
* & * & * & * & Q_{\dot{\xi}} & 0 \\
* & * & * & * & * & Q_{d}
\end{array}\right]\left[\begin{array}{c}
e_{1} \\
e_{22} \\
e_{\xi} \\
\xi \\
\dot{\xi} \\
d
\end{array}\right] \leq 1 .
$$

Let $\zeta=\operatorname{col}\left(e_{1}, e_{22}, e_{\xi}, \xi, \dot{\xi}, d\right)$. Define

$$
\begin{aligned}
& A_{00}:=\left[\begin{array}{cccccc}
\Xi_{11} & \Xi_{12} & 0 & 0 & 0 & \Xi_{16} \\
* & \Xi_{22} & 0 & 0 & 0 & \Xi_{26} \\
* & * & -2 \sigma I & \sigma I & \Gamma^{-1} & 0 \\
* & * & * & 0 & 0 & 0 \\
* & * & * & * & 0 & 0 \\
* & * & * & * & * & 0
\end{array}\right], \\
& A_{01}:=\left[\begin{array}{cccccc}
-P_{1} & 0 & 0 & 0 & 0 & 0 \\
* & -P_{22} & 0 & 0 & 0 & 0 \\
* & * & -\Gamma^{-1} & 0 & 0 & 0 \\
* & * & * & 0 & 0 & 0 \\
* & * & * & * & 0 & 0 \\
* & * & * & * & * & 0
\end{array}\right], A_{02}:=\left[\begin{array}{cccccc}
0 & 0 & 0 & 0 & 0 & 0 \\
* & 0 & 0 & 0 & 0 & 0 \\
* & * & 0 & 0 & 0 & 0 \\
* & * & * & \frac{1}{\alpha d_{0}} Q_{\xi} & 0 & 0 \\
* & * & * & * & \frac{1}{\alpha d_{0}} Q_{\xi} & 0 \\
* & * & * & * & \frac{1}{\alpha d_{0}} Q_{d}
\end{array}\right]
\end{aligned}
$$

and $f_{0 i}(\zeta):=\zeta^{T} A_{0 i} \zeta$.

According to the S-procedure, the inequalities $f_{01}(\zeta) \leq-1$ and $f_{02}(\zeta) \leq 1$ imply $f_{0}(\zeta)<0$ if and only if there exist $\tau_{01}, \tau_{02} \geq 0$ such that $A_{00}<\tau_{01} A_{01}+\tau_{02} A_{02}$ and $0 \geq-\tau_{01}+\tau_{02}$.

Based on Chetaev's instability theorem presented in [14], it can be obtained that if $\dot{V}>0$ for any $\left(e_{1}, e_{22}, e_{\xi}\right) \in \varepsilon(\mathscr{P})$ and 
for any $\operatorname{col}(\xi, \dot{\xi}, d)$ satisfying Assumption 1 , then $\left(e_{1}, e_{22}, e_{\xi}\right) \in$ $\varepsilon(\mathscr{P})$ will escape the invariant ellipsoid $\varepsilon(\mathscr{P})$ in finite time.

Define

$$
\begin{aligned}
\text { Define } & A_{10}:=\left[\begin{array}{cccccc}
-\Xi_{11} & -\Xi_{12} & 0 & 0 & 0 & -\Xi_{16} \\
* & -\Xi_{22} & 0 & 0 & 0 & -\Xi_{26} \\
* & * & 2 \sigma I & -\sigma I & -\Gamma^{-1} & 0 \\
* & * & * & 0 & 0 & 0 \\
* & * & * & * & 0 & 0 \\
* & * & * & * & * & 0
\end{array}\right], \\
A_{11}:= & {\left[\begin{array}{ccccccccc}
P_{1} & 0 & 0 & 0 & 0 & 0 \\
* & P_{22} & 0 & 0 & 0 & 0 \\
* & * & \Gamma^{-1} & 0 & 0 & 0 \\
* & * & * & 0 & 0 & 0 \\
* & * & * & * & 0 & 0 \\
* & * & * & * & * & 0
\end{array}\right], A_{12}:=\left[\begin{array}{ccccccc}
0 & 0 & 0 & 0 & 0 & 0 \\
* & 0 & 0 & 0 & 0 & 0 \\
* & * & 0 & 0 & 0 & 0 \\
* & * & * & -\frac{1}{\beta d_{1}} Q_{\xi} & 0 & 0 \\
* & * & * & -\frac{1}{\beta d_{1}} Q_{\xi} & 0 \\
* * & * & * & * & -\frac{1}{\beta d_{1}} Q_{d}
\end{array}\right] }
\end{aligned}
$$

and $f_{1 i}(\zeta):=\zeta^{T} A_{1 i} \zeta$.

It follows from Assumptions 1 that

$$
\xi^{T} Q_{\xi} \xi+\dot{\xi}^{T} Q_{\xi} \dot{\xi}+d^{T} Q_{d} d \geq \beta d_{1} .
$$

According to the S-procedure, the inequalities $f_{11}(\zeta) \leq 1$ and $f_{12}(\zeta) \leq-1$ imply $f_{10}(\zeta)<0$ if and only if there exist $\tau_{11}, \tau_{12} \geq$ 0 such that $A_{10}<\tau_{11} A_{11}-\tau_{12} A_{12}$ and $0 \geq \tau_{11}-\tau_{12}$.

Consider a sliding surface

$$
\mathcal{S}=\left\{\operatorname{col}\left(e_{1}, e_{21}, e_{22}, e_{\xi}\right) \mid e_{21}=0\right\} .
$$

Then the following result is ready to be presented.

Proposition 2: Under Assumptions 1, before the fault signal develops to incipient fault degree, the error system (27)-(30) is driven to sliding surface $\mathcal{S}$ given by (40) in finite time and remains on it thereafter, if the gain $\rho(\cdot)$ in $(26)$ is chosen to satisfying

$$
\rho(\cdot) \geq\left\|A_{a 211}(\omega)\right\| \sqrt{\frac{1}{\lambda_{\min }(\mathscr{P})}}+\left\|E_{a 21}\right\| \sqrt{\frac{1}{\lambda_{\min }\left(Q_{d}\right)}}+\eta
$$

where $\lambda_{\min }(\mathscr{P})$ and $\lambda_{\min }\left(Q_{d}\right)$ represents the minimum eigenvalue of $\mathscr{P}$ and $Q_{d}$ respectively, and $\eta$ is any positive scalar.

Proof: It follows from Proposition 1 that before the fault signal develops to incipient fault degree $\operatorname{col}\left(e_{1}, e_{22}, e_{\xi}\right) \in \varepsilon(\mathscr{P})$. Then $\left\|e_{1}\right\| \leq \sqrt{\frac{1}{\lambda_{\min }(\mathscr{P})}}$. Moreover, it follows from Assumption 1 that $\|d\| \leq \sqrt{\frac{1}{\lambda_{\min }\left(Q_{d}\right)}}$. A Lyapunov candidate function $V=e_{21}^{T} e_{21}$ is chosen for error sub-system (28). Since $G_{21}$ is symmetric positive definite and $\rho(\cdot)$ is chosen to satisfy (41), it can yield that $\dot{V} \leq-\eta V^{\frac{1}{2}}$. Thus, the $\eta$-reachability condition is satisfied, which implies that the error system (27)-(30) is driven to sliding surface $\mathcal{S}$ in (40) in finite time and remains on it thereafter. Hence, the result follows.

\section{Incipient Fault Detection Decisions}

Firstly, the incipient fault detection residual in this paper is defined by

$$
r=\operatorname{col}\left(e_{1}, e_{22}, e_{\xi}\right)
$$

Then, the threshold is chosen as $\varepsilon(\mathscr{P})$. In this paper, the following logical relationships are used for incipient fault detection

$$
\begin{aligned}
& r \in \varepsilon(\mathscr{P}) \Rightarrow \text { no alarm, faults free, } \\
& r \notin \varepsilon(\mathscr{P}) \Rightarrow \text { alarm, incipient faults are detected. }
\end{aligned}
$$

Therefore, for the incipient faults occurring after sliding motion taking place, the decision on the occurrence is made if the residual $r$ exceeds the threshold $\varepsilon(\mathscr{P})$. The detection time instant $T_{d}$ is defined as the first time instant when $r \notin \varepsilon(\mathscr{P})$.

Then the following theorem is ready to be presented.

Theorem 1: For the inverter sensor faulty system (3) and incipient sensor faults modeled by (5) with fault parameters $\xi$, $\dot{\xi}$ and disturbances satisfying Assumptions 1, the fault detection decision scheme characterized by observer (21)-(25), residual $r$ defined in (42) and threshold $\varepsilon(\mathscr{P})$ guarantees that there is no false alarm before incipient faults occur, and that there is no missing alarm for the incipient faults satisfying Assumption 1 .

\section{Simulation}

To verify the effectiveness of the proposed method, the case that two incipient voltage sensor faults occur simultaneously is considered. The practical parameters of the three-phase PWM inverter in CRH2 from CRRC ZHUZHOU INSTITUTECO., LTD are provided in the following table. When two incipient

TABLE I

Parameters of the inverter in CRH2.

\begin{tabular}{lcc}
\hline \hline Parameter & Value & Unit \\
\hline$r$ & 0.144 & $\Omega$ \\
$L_{f}$ & $1.417 \times 10^{-3}$ & $H$ \\
$C_{f}$ & $6000 \times 10^{-6}$ & $F$ \\
$V_{d c}$ & 3600 & $V$ \\
\hline \hline
\end{tabular}

faults occur on the $v_{o d}$ and $v_{o q}$ voltage sensors simultaneously, the fault distribution matrix $F$ is described by

$$
F=\left[\begin{array}{ll}
1 & 0 \\
0 & 1
\end{array}\right]
$$

The incipient sensor faults are assumed to be generated by

$$
\dot{f}=\left[\begin{array}{cc}
-100 & \\
20 & -100
\end{array}\right] f+\left[\begin{array}{l}
\xi_{1} \\
\xi_{2}
\end{array}\right], f(0)=0
$$

where

$$
\begin{aligned}
& \xi_{1}= \begin{cases}0, & 0 \leq t<1 s, \\
2000 \sin (3 t), & 1 s \leq t,\end{cases} \\
& \xi_{2}= \begin{cases}0, & 0 \leq t<1 s, \\
1000 \sin (3 t), & 1 s \leq t .\end{cases}
\end{aligned}
$$

The bounded noise is given by

$$
d=\left[\begin{array}{l}
1.5 \sin (10 t) \\
2.0 \sin (10 t)
\end{array}\right] \text {. }
$$

Thus, $Q_{\xi}, Q_{\xi}, Q_{d}, \Gamma_{0}, \underline{\Gamma}, \bar{\Gamma}$ and $d_{0}, d_{1}$ in Assumption 1 can be obtained, and the augmented system can be established as (6).

It can be verified that the augmented system satisfies Assumption 1 . The parameters in detection observer are calculated based on Proposition 1 and 2. The estimation results are shown in Figs. 3-6. 
It can be seen from Figs. 3, 5 and 6 that the residual $r$ defined in (42) converges to the designed invariant ellipsoid before the sensor fault develops to incipient fault degree. It is clear from Fig. 4 that the sliding motion takes place infinite time and maintains on the sliding surface thereafter.

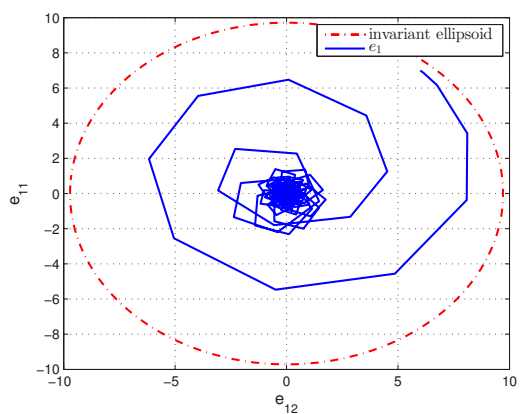

Fig. 3. Time response of $e_{1}$ and corresponding invariant ellipsoid.
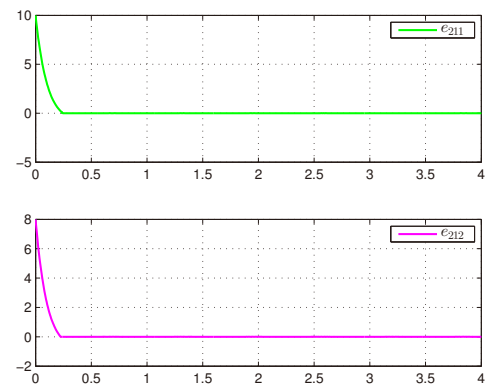

Fig. 4. Time response of $e_{21}$ and corresponding invariant ellipsoid.

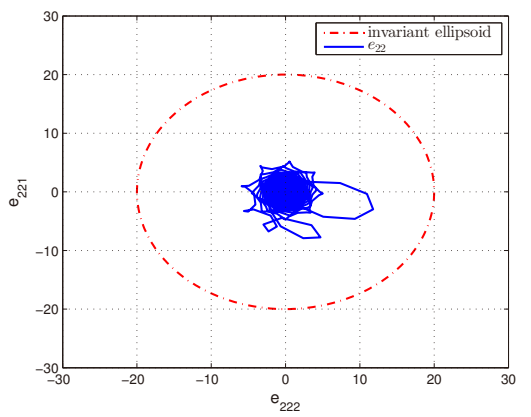

Fig. 5. Time response of $e_{22}$ and corresponding invariant ellipsoid.

From Fig. 6, it can be seen that the estimation error $e_{\xi}$ which is one part of the residual $r$ starts in the invariant ellipsoid and converges to the area before the sensor faults develop to the incipient fault degree. With the development of the sensor faults, the faults develop to incipient fault degree at $T_{0}$, then the estimation error $e_{\xi}$ diverges out the invariant ellipsoid at $T_{d}$ and stay outside of the ellipsoid. Thus, based on the incipient fault decision principle, the incipient fault is detected at time instant $T_{d}$.

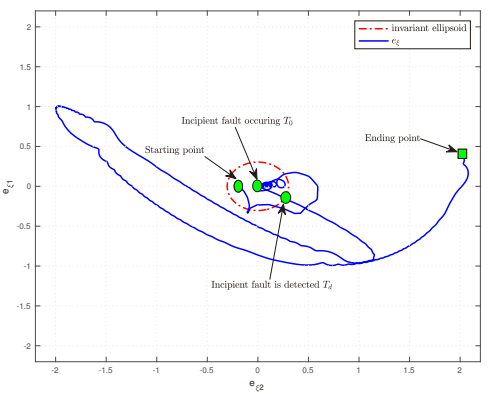

Fig. 6. Time response of $e_{\xi}$ and corresponding invariant ellipsoid.

\section{Conclusion}

This paper has proposed an adaptive and sliding mode unknown input estimation modules as sensor incipient fault detection observer for the inverter device in high-speed railway traction systems. The novel method to distinguish incipient faults from disturbances has been provided. Based on this distinguish method, the invariant ellipsoid concept has been introduced and particularly designed as threshold to detect the sensor incipient faults. The simulation results has been presented to verify the effectiveness and practicalness of the proposed incipient fault detection schemes.

\section{REFERENCES}

[1] Brenna M, Foiadelli F. Analysis of the filters installed in the interconnection points between different railway supply systems. IEEE Transactions on Smart Grid 2012; 3(1): 551-558.

[2] Bae C, Jang D, Kim Y. Calculation of regenerative energy in DC $1500 \mathrm{~V}$ electric railway substations. 7th International IEEE Conference on Power Electronics 2007; 801-805.

[3] Youssef AB, Khil E, Khojet S. State observer-based sensor fault detection and isolation, and fault-tolerant control of a single-phase PWM rectifier for electric railway traction. IEEE Transactions on Power Electronics 2013; 28(12): 5842-5853.

[4] Jiang B, Staroswiecki M, Cocquempot V. Fault accommodation for nonlinear dynamic systems. IEEE Transactions on Automatic Control 2006; 51(9): 1578

[5] X. Zhang, Sensor bias fault detection and isolation in a class of nonlinear uncertain systems using adaptive estimation, IEEE Transactions on Automatic Control, 2011; 56(5): 1220-1226.

[6] Edwards C, Spurgeon SK, Patton RJ. Sliding mode observers for fault detection and isolation. Automatica 2000; 36(4): 541-553.

[7] Yan XG, Edwards C. Nonlinear robust fault reconstruction and estimation using a sliding mode observer. Automatica 2007; 43(9): 1605-1614.

[8] Nazin S A, Polyak B T, Topunov M V. Rejection of bounded exogenous disturbances by the method of invariant ellipsoids. Automation and Remote Control, 2007, 68(3): 467-486.

[9] Polyakov A, Poznyak A. Invariant ellipsoid method for minimization of unmatched disturbances effects in sliding mode control. Automatica, 2011, 47(7): 1450-1454.

[10] Zhang K, Jiang B, Yan X G, et al. Incipient sensor fault estimation and accommodation for inverter devices in electric railway traction systems. International Journal of Adaptive Control and Signal Processing, 2016.

[11] Saif M, Guan Y. A new approach to robust fault detection and identification. IEEE Transactions on Aerospace and Electronic Systems 1993; 29(3): 685-695.

[12] Edwards C, Yan XG, Spurgeon SK. On the solvability of the constrained Lyapunov problem. IEEE Transactions on Automatic Control 2007; 52(10): 1982-1987.

[13] Khalil H K. Nonlinear Systems. Prentice, Hall, New Jersey, 1996. 